\title{
Qualidade de Vida e Bem-Estar Subjetivo de Sobreviventes ao Câncer Ósseo: Percepção de Sobreviventes e Familiares
}

\author{
Juliana Fákir Naves" \\ Rede SARAH de Hospitais, Brasília, DF, Brasil \\ Tereza Cristina Cavalcanti Ferreira de Araujo* \\ Universidade de Brasília, Brasília, DF, Brasil
}

\begin{abstract}
RESUMO
Em Psico-Oncologia, é crescente o interesse por estudos que fundamentem programas eficazes destinados à etapa de sobrevivência. Destarte, objetivou-se conhecer e analisar a qualidade de vida e o bem-estar subjetivo de sobreviventes, com diagnóstico anterior de câncer ósseo. Para tanto, 27 sobreviventes e 25 familiares preencheram os seguintes instrumentos: Questionário de Avaliação da Experiência Oncológica do Sobrevivente, Escala de Qualidade de Vida para Sobreviventes de Câncer e Escala de Bem-Estar Subjetivo. A percepção de qualidade de vida dos sobreviventes revelou-se mais positiva do que para seus familiares. O domínio psicológico foi estimado como de pior desempenho. A percepção de bem-estar subjetivo foi semelhante para sobreviventes e familiares. Não houve diferença quanto à idade, localização do tumor e renda entre sobreviventes.
\end{abstract}

Palavras-chave: qualidade de vida; bem-estar subjetivo; câncer; sobrevivência.

\section{ABSTRACT \\ Quality of Life and Subjective Well-Being of Bone Cancer Survivors: Perception of Survivors and Family}

In Psycho-Oncology, there is growing interest in studies that substantiate effective programs for the survival stage. Thus, the objective was to understand and analyze the quality of life and subjective well-being of survivors, with previous diagnosis of bone cancer. For this, 27 survivors and 25 families' members completed the following instruments: Oncological Experience Assessment for Survivors, Quality of Life for Cancer Survivors and Scale of Subjective Well-Being. The perceived quality of life of survivors was more positive than for their relatives. The psychological domain was estimated as the worst performer. The perception of subjective well-being was similar for survivors and families. There were no differences in age, tumor location and income among survivors.

Keywords: quality of life; subjective well-being; cancer; survival.

Inicialmente, estudos destinados à avaliação do impacto do câncer sobre a qualidade de vida focalizaram as etapas diagnóstica e terapêutica. Com a redução dos óbitos e o prolongamento da vida dos pacientes, intensificaram-se as pesquisas acerca das consequências físicas e psicossociais durante a etapa designada, em Oncologia, como sobrevivência. Cumpre esclarecer que embora existam avanços nessa área, efeitos colaterais ocasionados pelos tratamentos podem ser irreversíveis, impondo intervenções suplementares e comprometendo a adaptação do indivíduo.
Mas, transformações satisfatórias também têm sido identificadas na vida dessas pessoas (Araujo \& Arrais, 1998; Holland \& Reznik, 2005; Pearce, SansonFisher, \& Campbell, 2008; Schwartz, Werba, \& Kazak, 2010; Stanton, 2010).

Ainda não existe consenso quanto ao conceito de sobrevivência relacionada ao câncer. Ao se examinar estudos sobre o assunto, encontra-se que um sobrevivente pode ser um indivíduo que: a) não está sob tratamento pelo menos há um ano; b) não apresenta sintomas há pelo menos cinco anos; ou c) não faleceu,

\footnotetext{
Endereço para correspondência: Juliana Fákir Naves - juliananaves@sarah.br

Endereço para correspondência: Tereza Cristina Cavalcanti Ferreira de Araujo - araujotc@unb.br
} 
decorridos cinco anos ou mais desde o diagnóstico (Barakat, Alderfer, \& Kazak, 2006; Boaventura \& Araujo, 2012; Clinton-McHarg, Carey, Sanson-Fisher, Shakeshaft, \& Rainbird, 2010). Frente a essa disparidade, muitos questionam a necessidade de se estabelecer critérios rígidos para definir sobrevivência, uma vez que o câncer é uma enfermidade complexa, que exige um contexto específico de cuidados, os quais delimitarão a experiência oncológica ao longo de todas as suas etapas. Nesse sentido, é interessante enfatizar que Khan et al., (2012) estendem o espectro conceitual e incluem familiares e cuidadores que participaram ativamente do acompanhamento do paciente na categoria de sobreviventes.

Atualmente, diante da amplitude do follow-up necessário para assegurar o alcance de metas preventivas e de promoção da saúde voltadas para sobreviventes oncológicos, defende-se a implementação de programas que articulem equipes de saúde multiprofissionais e serviços comunitários (Demark-Wahnefried, 2010; Ganz \& Hahn; 2010; Perina, Mastellaro, \& Nucci, 2008; Tai et al., 2012). Para tanto, estimula-se a realização de mais estudos sobre o tema, no intuito de fundamentar ações eficazes desde a atenção básica até serviços especializados nessa área.

De fato, em Psico-Oncologia, inúmeros trabalhos foram empreendidos com o propósito de avaliar as modificações produzidas nos diferentes domínios da existência após diagnóstico de um câncer e intervenção terapêutica. No tocante à qualidade de vida, desde a década de 1930, conceituações e instrumentos de medida diversificados foram propostos, sobressaindose a natureza multidimensional, dinâmica e subjetiva do constructo (Costa Neto \& Araujo, 2008; DelgadoSanz et al., 2011; Morganstern, Bochner, Dalbagni, Shabsigh, \& Rapkin, 2011; Nucci \& Valle, 2006; Santos, Moura, Santos, Figueiroa, \& Souza, 2012). Vale lembrar que para a Organização Mundial de Saúde (OMS), qualidade de vida corresponde à "percepção do indivíduo de sua posição na vida, no contexto de sua cultura e no sistema de valores em que vive e em relação a suas expectativas, seus padrões e suas preocupações" (WHOQOL Group, conforme citado por Fleck, 2008, p. 25). Por conseguinte, a avaliação da qualidade de vida resulta da percepção positiva ou negativa do indivíduo sobre vários domínios que pode ou não ser afetada pelas condições de saúde ou pelo modo como ele percebe sua saúde. Constitui-se em um indicativo sobre o impacto do estado de saúde na capacidade de viver plenamente, mas não corresponde à descrição do status de saúde de uma pessoa (Fleck, 2008; Patrick, 2008; Silva \& Derchain, 2006).

Paralelamente, nas últimas décadas, o bem-estar subjetivo tem suscitado interesse entre pesquisadores, inclusive no Brasil. Assim como ocorre com a qualidade de vida, não existe consenso quanto a sua delimitação conceitual. Pode ser considerado como uma medida global de estado que se modifica periodicamente em função de mecanismos internos e da interação com o mundo, sendo que as circunstâncias externas influenciam, mas não são fatores constituintes do bem-estar. Não estaria relacionado apenas à ausência de fatores negativos, mas principalmente à presença de fatores positivos (Albuquerque \& Tróccoli, 2004; Chandra et al., 1998; Costa \& Pereira, 2007; Giacomoni, 2004; Siqueira \& Padovam, 2008). Visando a avaliação do bem-estar subjetivo, diferentes instrumentos foram elaborados e adotados em pesquisas internacionais e nacionais, como por exemplo: Index de Bem-Estar (Campbell, Converse, \& Rodgers, 1976, conforme citado por Giacomini, 2004); Escala de Satisfação com a Vida (Diener, Emmons, Larsen, \& Griffin, 1985); Escala de Bem-Estar Subjetivo (Lawrence \& Liang, 1988); Escala de Afeto Positivo e Afeto Negativo (Watson, Clark, \& Tellegen, 1988); Escala de Bem-estar Subjetivo (Albuquerque \& Tróccoli, 1994) e Escala de Ânimo Positivo e Negativo (Siqueira et al., 1999, conforme citado por Giacomini, 2004). Em saúde, algumas pesquisas têm almejado conhecer o bem-estar subjetivo de indivíduos acometidos por cronicidades.

Ao se realizar um levantamento dos trabalhos sobre qualidade de vida e bem-estar subjetivo relacionados à saúde - mais precisamente aqueles aplicados ao contexto oncológico - é possível constatar predomínio da primeira temática, apesar da necessidade de mais estudos sobre a concepção e a satisfação do indivíduo no que diz respeito ao seu bem-estar. Em síntese, um exame atento da literatura permite reconhecer uma interface conceitual, a qual impõe o desenvolvimento de estudos abrangentes que contemplem ambos os enfoques (Chandra et al., 1998; Costa \& Pereira, 2007; Giacomoni, 2004; Siqueira \& Padovam, 2008).

No que se refere aos cânceres ósseos - e especialmente às vivências associadas à sobrevivência - são escassas as pesquisas destinadas a aprofundar conhe- 
cimentos teóricos e práticos. Cabe esclarecer que tumores ósseos têm maior incidência na população jovem com menos de 20 de idade. Nessa categoria diagnóstica, incluem-se sobretudo o osteossarcoma e o sarcoma de Ewing (Klosky \& Spunt, 2010; Petrilli, 2008). Nos Estados Unidos, dados epidemiológicos mostraram 6,5 pacientes por milhão de crianças ou 900 casos novos a cada ano. No Brasil, não existem estimativas atuais relativas aos tumores ósseos, mas o Instituto Nacional do Câncer divulgou que, entre 1967 a 2009, foram diagnosticados 669 casos de tumores ósseos em crianças e adolescentes em 20 cidades brasileiras (Bispo Júnior \& Camargo, 2011; INCA, 2011).

Vale explicitar que casos de câncer ósseo exigem hospitalizações prolongadas e frequentes, exames invasivos, cirurgias (por exemplo, amputação), tratamento quimioterápico com efeitos colaterais e sequelas provocadas pelas próprias intervenções. De acordo com Eiser (2009), as taxas de sobrevida são menores quando comparadas às de outros tipos de câncer, sendo que um prognóstico mais favorável depende do tamanho do tumor, sua localização, possibilidade de remoção cirúrgica e ausência de metástases. Sintomas como fadiga crônica, menopausa precoce, dor, disfunções cardíacas ou renais, distúrbios de fertilidade e alterações do sono são relatados por sobreviventes (Petrilli, 2008). Perina, Mastellaro e Nucci (2008) citam o trabalho de Oeffinger et al. (2006), no qual três quartos de uma amostra de 10.397 sobreviventes comunicaram algum problema crônico de saúde após 30 anos.

Sendo assim, é válido supor que a existência de sintomas e sequelas resulta em prejuízos para a qualidade de vida e o bem-estar desses indivíduos. Estudos anteriormente conduzidos apontaram indicadores piores, sobretudo no domínio físico de sobreviventes de osteossarcoma, os quais experimentam mais limitações funcionais e dor (Barr \& Wunder, 2009; Eiser, 2009; Maurice-Stam et al., 2006). Mas, a complexidade da problemática estudada revela-se quando se verifica que o empobrecimento do domínio físico não se correlaciona com a qualidade de vida global (Ness, Bhaskar, Rao, \& Daw, 2009; Yonemoto et al., 2007). Vale dizer que, segundo Koopman et al. (2005) e Eiser (2009), os sobreviventes desse tipo de câncer têm uma qualidade de vida comparável à da população geral.
Adicionalmente, é interessante assinalar que relatos de melhoria em aspectos da qualidade de vida de pacientes com osteossarcoma, poucas semanas após o início do tratamento, tenderam a diminuir com o passar do tempo (Morganstern et al., 2011). Um estudo longitudinal indicou que embora ocorram ganhos em habilidade funcional e no nível de atividade física, isso não foi observado em relação ao domínio psicológico no período de até um ano após a cirurgia (Bekkering et al., 2012).

Cumpre notar ainda que indivíduos diagnosticados na vida adulta apresentaram pior desempenho da saúde física, mas seus escores quanto à qualidade de vida e saúde mental revelaram-se positivos. Mulheres com câncer ósseo tenderiam a perceber sua qualidade de vida como pior do que homens, a exemplo do que já se observou entre mulheres e homens da população em geral. Quando comparados com seus irmãos, sobreviventes de osteossarcoma apresentaram déficits em educação, empregabilidade e facilidade para adquirir seguro de saúde, sendo que um alto nível educacional foi considerado bom preditor para empregabilidade futura (Eiser, 2009; Nagarajan et al., 2003; Nagarajan et al., 2011; Zebrack et al., 2008).

Tendo em vista que sobreviventes de câncer ósseo submetidos à amputação enfrentam deformidades estéticas com consequências psicossociais, é importante ponderar as dificuldades desencadeadas para indivíduos adolescentes e jovens, em razão da busca por integração em um grupo social, a qual caracteriza esse período do desenvolvimento humano (Ness et al., 2009; Perina et al., 2008). No entanto, não se constatou relação entre o tipo de cirurgia (conservadora do membro afetado ou de amputação) e a percepção de qualidade de vida após ressecção do tumor ósseo (Barr \& Wunder, 2009; Frances, Morris, Arkader, Nikolic, \& Healey, 2007). Furst e Humphrey (1983) alertam que terceiros podem avaliar as dificuldades decorrentes de uma amputação como mais restritivas em comparação à avaliação feita pelas pessoas amputadas.

Possivelmente, as divergências entre estudos que visam compreender a sobrevivência derivam da heterogeneidade tanto das amostras pesquisadas, como das medidas adotadas (Araujo \& Arrais, 1998; Frances et al., 2007). Torna-se imprescindível, então, empregar instrumentos específicos construídos para sobreviventes (Maurice-Stam et al., 2009), como o Quality of 
Life for Cancer Survivors (QOL-CS), desenvolvido por Grant, Padilla e Ferrell e modificado ulteriormente por Ferrell e Hassey-Dow (Ersek, Ferrell, HasseyDow, \& Melancon, 1997). Do ponto de vista de Nagarajan et al. (2004) e Pearce et al. (2008), esse instrumento possui características psicométricas satisfatórias - como confiabilidade, replicabilidade, viabilidade, aceitabilidade e validade preditiva - que recomendam seu uso em pesquisas, a exemplo daquelas já conduzidas por Ahles et al. (2005) e Chopra e Kamal (2012).

Considerando, portanto, os interesses assistenciais e científicos discutidos anteriormente, empreendeu-se uma investigação com os seguintes objetivos: a) conhecer e analisar a percepção de qualidade de vida e de bem-estar subjetivo de sobreviventes de câncer ósseo; b) conhecer e analisar a percepção dos familiares acerca da qualidade de vida e do bem-estar subjetivo dos sobreviventes; e c) comparar as percepções de ambos os segmentos estudados.

\section{MÉTODO}

\section{Participantes}

Preliminarmente, foi realizado um levantamento dos pacientes admitidos no Programa de Oncologia da Rede SARAH com auxílio do setor de Controle de Qualidade. Em seguida, estabeleceram-se como critérios de inclusão na amostra: indivíduos anteriormente tratados no serviço, em razão de um diagnóstico de câncer ósseo do tipo osteossarcoma ou sarcoma de Ewing, com idade igual ou superior a 18 anos, e que haviam recebido alta do programa por estarem pelo menos há cinco anos em remissão da doença. Foram excluídos sujeitos com alteração cognitiva ou neuropsicológica que constituíssem impedimento para o preenchimento autônomo dos instrumentos propostos. A subamostra de familiares foi composta por um membro da família, indicado pelo próprio sobrevivente, que tinha contato direto ou fosse considerado próximo. Nesta pesquisa, os familiares não serão incluídos na amostra de sobreviventes, constituindo uma categoria distinta.

\section{Instrumentos}

- Questionário de Avaliação da Experiência Oncológica do Sobrevivente: elaborado para obtenção de dados sociodemográficos e de informações sobre a percepção das experiências re- lacionadas ao câncer e suas consequências. Foi preenchido apenas pelo sobrevivente.

- Escala de Qualidade de Vida para Sobreviventes de Câncer (EQV-SC): versão traduzida e adaptada da Quality of Life Cancer Survivors (QOL-CS), desenvolvida especialmente para esta pesquisa. O instrumento original é composto por 41 itens, abrangendo os domínios bemestar físico, bem-estar psicológico, preocupações sociais e bem-estar espiritual, avaliados por meio de uma escala do tipo Likert, de dez pontos.

- Escala de Bem-Estar Subjetivo (EBES): o instrumento proposto por Albuquerque e Tróccoli (2004) é composto por duas subescalas. Para a presente investigação, adotou-se apenas a subescala destinada à avaliação de sentimentos e emoções, ponderados como positivos ou negativos a partir de uma escala Likert de cinco pontos, empregando-se a média obtida. Foi realizada uma adaptação do layout desta subescala para apresentação aos participantes.

É necessário explicitar que os familiares responderam aos instrumentos de qualidade de vida e de bemestar, tomando como base a percepção que possuíam acerca das vivências do sobrevivente e não aquelas decorrentes de suas próprias experiências.

\section{Procedimentos para coleta e análise de dados}

O projeto foi previamente autorizado por Comitê de Ética em Pesquisa. Um convite inicial foi feito por contato telefônico. Em seguida, foram enviados, por correio ou e-mail, os instrumentos mencionados no item anterior, devidamente apensados de instruções para preenchimento e do Termo de Consentimento Livre e Esclarecido (TCLE). Os dados obtidos pela Escala de Qualidade de Vida (EQV-SC) e pela Escala de Bem-Estar Subjetivo (EBES) foram analisados estatisticamente por meio do software Statistical Package for the Social Sciences (SPSS 19.0). Foram realizadas comparações por meio dos testes de Wilcoxon e de Mann-Whitney.

\section{RESULTADOS E DISCUSSÃO}

A Tabela 1 apresenta a caracterização sociodemográfica dos sobreviventes estudados. Constata-se que a maioria havia recebido tratamento oncológico em 
razão de um osteosarcoma. Quanto à localização do tumor, verifica-se que o fêmur foi o sítio primário de câncer mais frequente $(n=18)$, seguido por tíbia $(n=$ $3)$ e fíbula $(n=2)$. Esses dados convergem com evi- dências assinaladas na literatura internacional que indicam predomínio de câncer em ossos longos em comparação com outras estruturas ósseas (Klosky \& Spunt, 2010; Nagarajan et al., 2004).

\begin{tabular}{|c|c|}
\hline \multicolumn{2}{|l|}{ Características } \\
\hline Sarcoma de Ewing & $n=5$ \\
\hline Osteossarcoma & $n=22$ \\
\hline Gênero & $\begin{array}{l}\text { Mulheres } n=13 \\
\text { Homens } n=14\end{array}$ \\
\hline Média de idade & $\begin{array}{l}17,4 \text { anos na época do diagnóstico } \\
29,9 \text { anos por ocasião da pesquisa }\end{array}$ \\
\hline Procedência & $\begin{array}{l}\text { Distrito Federal: } 51,9 \% \\
\text { Outras regiões: } 49,1 \%\end{array}$ \\
\hline Formação acadêmica & $\begin{array}{l}\text { Ensino fundamental: } 15 \% \\
\text { Ensino médio: } 37 \% \\
\text { Ensino superior: } 44 \% \\
\text { Pós-Graduação: } 4 \%\end{array}$ \\
\hline Em atividade laboral & $\begin{array}{l}\text { Sim: } 66,7 \% \\
\text { Não: } 33,3 \%\end{array}$ \\
\hline Amputação & $\begin{array}{l}\text { Sim: } 74,1 \% \\
\text { Não: } 25,9 \%\end{array}$ \\
\hline Sítio primário & $\begin{array}{l}\text { Fêmur: } 66,67 \% \\
\text { Tíbia: } 11,12 \% \\
\text { Fíbula: } 7,41 \% \\
\text { Úmero: } 3,70 \% \\
\text { Escápula: 3,70\% } \\
\text { Sacro: } 3,70 \% \\
\text { Hálux: 3,70\% }\end{array}$ \\
\hline Tempo de alta & $\begin{array}{l}\text { 1-5 anos: } 11 \% \\
\text { 6-10 anos: } 41 \% \\
\text { 11-15 anos: } 37 \% \\
21-25 \text { anos: } 4 \% \\
\text { Não mencionou: } 7 \%\end{array}$ \\
\hline Percepção da deficiência & $\begin{array}{l}\text { Sem deficiência: } 7,41 \% \\
\text { Leve: } 44,44 \% \\
\text { Moderada: } 40,74 \% \\
\text { Grave: } 7,41 \%\end{array}$ \\
\hline Uso de auxílio-locomoção & $\begin{array}{l}\text { 44\%: sem auxílio } \\
\text { 30\%: com auxílio } \\
\text { 15\%: uma bengala/muleta } \\
\text { 7\%: não respondeu } \\
\text { 4\%: andador }\end{array}$ \\
\hline Acompanhante no tratamento & $\begin{array}{l}\text { Sim: } 92 \% \\
\text { Não: } 8 \%\end{array}$ \\
\hline
\end{tabular}

$\mathrm{O}$ alto índice ocupacional dos sobreviventes $(66,7 \%)$ pode ser compreendido pelo maior nível educacional da amostra, o que também foi identificado Interação Psicol., Curitiba, v. 19, n. 3, p. 351-363, set./dez. 2015 por Nagarajan et al. (2003). Poucos participantes associaram mudanças em relação ao trabalho provocadas pela enfermidade. Possivelmente, isso se deve ao 
fato de não estarem empregados no início da experiência oncológica, em função da pouca idade por ocasião do diagnóstico. Apenas um sobrevivente relatou dificuldade relacionada ao trabalho.

A maior parte dos sobreviventes (92\%) contou com a presença de pelo menos um acompanhante durante o tratamento, em especial a mãe e os irmãos, e quando questionados acerca de aspectos positivos percebidos durante o tratamento, o principal fator comunicado foi suporte familiar $(n=19)$, seguido por apoio espiritual ou religioso $(n=9)$. No que tange às dificuldades enfrentadas, observa-se que as respostas mais frequentes se relacionaram com os efeitos colaterais da quimioterapia, em especial náusea $(n=8) \mathrm{e}$ dificuldade de se alimentar $(n=7)$. Estes resultados corroboram estudos que citam a quimioterapia como fator negativo mesmo após o fim do tratamento, podendo inclusive estar associada a uma pior qualidade de vida (Maurice-Stam et al., 2009).
Tal como esperado, as dificuldades atuais dos sobreviventes se concentram em habilidade físicafuncional: correr $(n=8)$, fazer caminhada ou andar apressadamente $(n=6)$ e praticar algum tipo de esporte $(n=5)$ (Frances et al., 2007; Maurice-Stam et al., 2006; Nagarajan et al., 2011).

As médias de qualidade de vida (geral) informadas por sobreviventes e familiares foram de 6,52 e 6,33, respectivamente. Esses resultados podem ser considerados satisfatórios e reafirmam os estudos de Eiser (2009), Koopman et al. (2005) e Maurice-Stam et al. (2009), nos quais se admite a capacidade do indivíduo para superar as adversidades suscitadas pelo câncer e seus tratamentos. A Figura 1 apresenta as médias de qualidade de vida atribuídas por sobreviventes e seus familiares aos diferentes domínios avaliados pela EQV-SC.

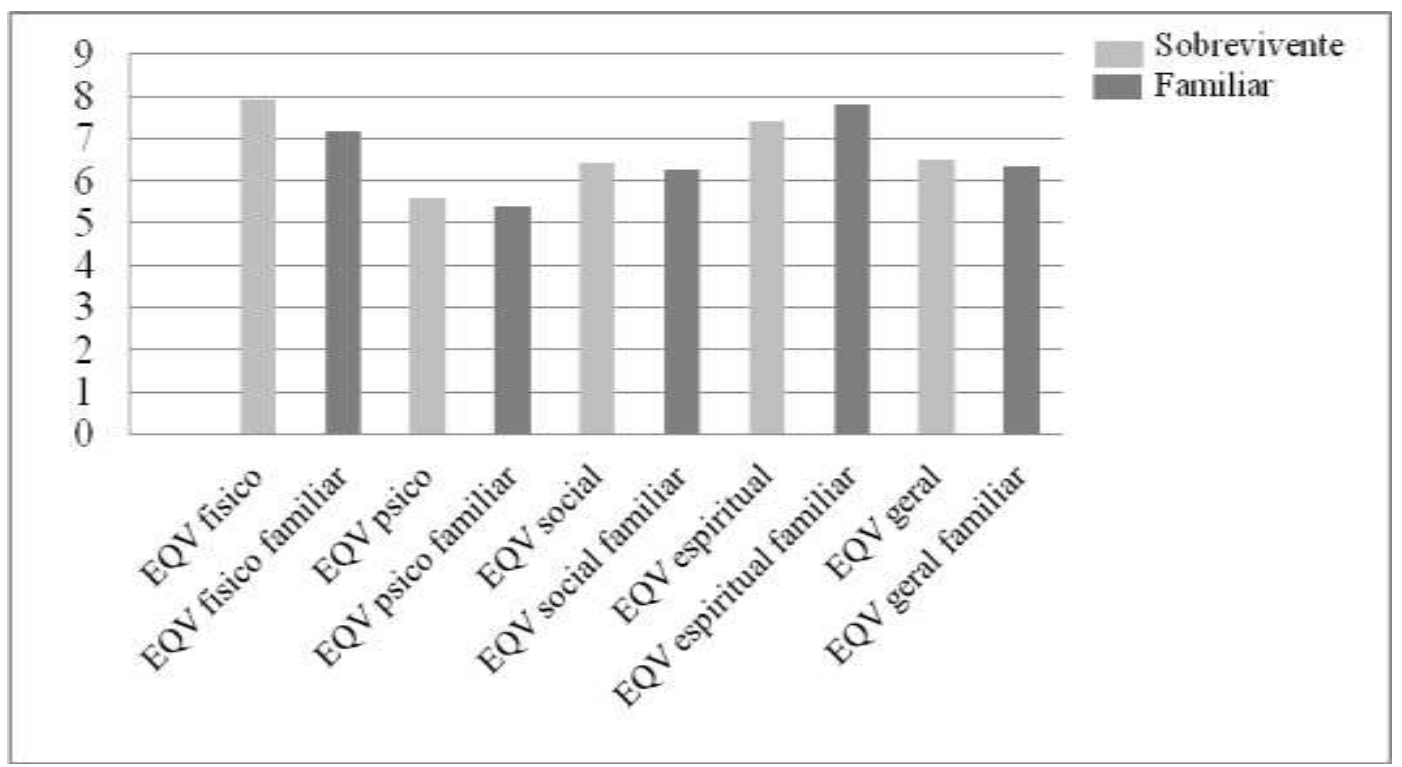

Figura 1. Comparação dos Domínios de Qualidade de Vida Avaliados por Sobreviventes e Familiares por meio da EQV-SC.

Ao se comparar os valores calculados, constata-se melhor percepção dos sobreviventes do domínio físico $(M=7,91)$, seguido pelo espiritual $(M=7,43)$. Para eles, o domínio psicológico é o de pior desempenho $(M=5,59)$. Tais evidências sugerem uma associação entre funcionamento físico e qualidade de vida geral (Maurice-Stam et al., 2006; Yonemoto et al., 2007). Já os familiares tenderam a perceber a capacidade física mais negativamente do que os sobreviventes $(p=$
0,02) e atribuíram melhores indicadores aos domínios espiritual $(M=7,80)$ e físico $(M=7,16)$. Igualmente para os familiares, o domínio psicológico foi considerado aquele de menor qualidade $(M=5,38)$.

Especificamente no domínio físico, o item de melhor desempenho de acordo com a ponderação feita por sobreviventes e seus familiares, foi saúde física em geral $(M=7,89)$. Entre os casos de amputação, a diferença entre sobreviventes e familiares foi estatisti- 
camente significativa ( $p=0,005 ; r=0,64)$, sendo que estes últimos tenderam a fazer uma avaliação mais desfavorável. No que concerne à diferença verificada, é importante lembrar que, segundo Furst e Humphrey (1983), outras pessoas avaliam o indivíduo amputado como tendo mais dificuldades do que ele mesmo reconhece. Isto alerta para a necessidade de se planejar e oferecer orientações voltadas especialmente para os familiares na fase cirúrgica.

Não houve diferença de percepção da qualidade de vida geral em relação à localização do tumor, mesmo para amputados $(p>0,05)$. Os escores relativos à percepção atual de dor $(M=3,37)$, fadiga $(M=2,22) \mathrm{e}$ alteração de sono $(M=1,78)$ mostraram-se baixos.

A pior avaliação no domínio psicológico, tanto para os sobreviventes quanto para familiares, contraria a literatura que tem apontado o domínio físico como mais limitado em casos de sobrevivência a um câncer ósseo. Assim, os resultados desta pesquisa, mesmo não apontando transtornos de saúde mental, parecem revelar dificuldades de ajustamento após o tratamento (Maurice-Stam et al., 2009; Zebrack et al., 2008). Cabe mencionar que, no estudo de Bekkering et al. (2012), verificaram-se ganhos em todos os domínios da qualidade de vida após a cirurgia para retirada do tumor, com exceção do domínio psicológico.

Nesse domínio específico, o nível de estresse associado ao tratamento $(M=9,70)$ e o nível de estresse vinculado ao diagnóstico $(M=9,30)$ apresentaram as maiores médias, o que converge com estudos anteriores que mostraram sintomas de estresse e até mesmo de estresse pós-traumático entre sobreviventes (Kazak et al., 2004). Em compensação, o baixo índice de sintomas depressivos encontrado exige prudência ao se vincular a experiência oncológica à depressão, uma vez que sintomas de ansiedade e depressão estão presentes na população em geral. Em outras palavras, tal evidência não está necessariamente relacionada à doença, mas pode ser compreendida em razão das dificuldades cotidianas intensificadas pelo câncer (Jagannathan \& Juvva, 2009). Chama atenção ainda que, para os familiares, o medo de um novo diagnóstico de câncer $(M=6,58)$ ou de uma recidiva $(M=5,96)$ foi ainda maior do que para os sobreviventes, a exemplo do que foi evidenciado por Salci e Marcon (2010).

Em relação ao domínio social, o nível de estresse que a doença gerou para a família apresentou a maior média $(M=9,19)$. Em contrapartida, tanto para sobreviventes quanto para familiares, a influência do tratamento na sexualidade $(M=1,85)$ e o isolamento social causado pela doença $(M=2,15)$ obtiveram as menores médias. As poucas mudanças percebidas em relação à sexualidade podem estar relacionadas à idade do sobrevivente por ocasião do diagnóstico e à dificuldade do familiar em avaliar questões relacionadas à intimidade de outrem.

No domínio espiritual, a percepção do quanto esperançoso o sobrevivente se sente atingiu a maior média $(M=9,04)$, seguida pelo quanto o indivíduo sente que há um propósito de vida $(M=8,93)$. Tal como apontado por Kim, Carver, Spillers, Crammer e Zhou (2011), os sobreviventes expressaram elevado bemestar espiritual e boa saúde física. O nível de incerteza sobre o futuro obteve menor média entre os sobreviventes $(M=4,41)$. Os familiares avaliaram o sobrevivente como esperançoso $(M=9,32)$, com propósito de vida $(M=9,08)$ e pouca incerteza em relação ao futuro $(M=4,92)$.

Não se observou diferença estatística na qualidade de vida percebida pelo sobrevivente em função da idade em que teve ciência do diagnóstico. Esse resultado assemelha-se àqueles reunidos por Nagarajan et al. (2003) e Zebrack et al. (2008), mas se contrapõe a Maurice-Stam et al. (2009), cujo trabalho afirma que crianças mais velhas tenderiam a perceber de forma mais negativa o tratamento e sua qualidade de vida. Não houve diferença para os demais domínios e para a qualidade de vida geral (QV geral: $p=0,03 ; r=0,54$ ).

A Figura 2 expõe resultados relativos ao bem-estar subjetivo de sobreviventes e familiares. É possível notar que a avaliação dos aspectos positivos da EBES mostrou-se similar para sobreviventes e familiares. 


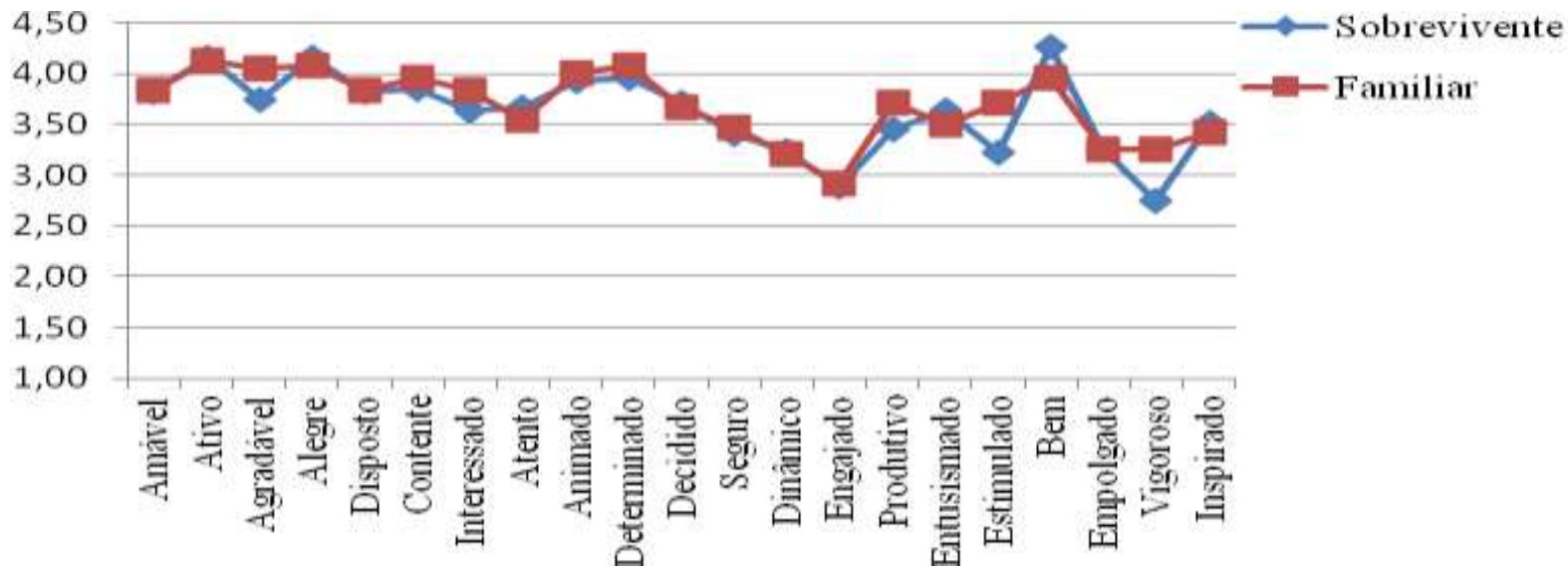

Figura 2. Relação entre Aspectos Positivos da EBES para Sobreviventes e Familiares.

Cabe salientar que foram encontradas fortes relações entre aspectos positivos da EBES e as subescalas EQV espiritual, EQV social e EQV geral $\left(r_{s}=0,71\right.$; $r_{s}=0,66 ; r_{s}=0,61$, respectivamente). A relação entre espiritualidade e saúde mental também foi evidenciada por Kim et al. (2011).

Verificaram-se fortes correlações negativas entre aspectos negativos da EBES e a EQV psicológica $\left(r_{s}=0,62\right)$, bem como relações moderadas entre EBES e EQV geral e EQV espiritual $\left(r_{s}=0,50 ; r_{s}=0,46\right)$. No domínio espiritual, constatou-se mais associação com aspectos positivos da EBES do que aspectos negativos. Não foi encontrada correlação estatisticamente significativa entre aspectos da EBES e a qualidade de vida estimada por familiares. Sendo assim, é possível supor que a qualidade de vida exerça influência sobre o bem-estar dos sobreviventes; mas isso não pode ser afirmado em relação aos familiares.

A Figura 3 ilustra os dados obtidos por homens e mulheres sobreviventes. Destaca-se que a percepção dos grupos masculino e feminino foi estatisticamente diferente no domínio físico da EQV, com pior percepção pelo gênero feminino. Esses dados corroboram trabalhos como os de Barr e Wunder (2009), Furst e Humphrey (1983), Nagarajan et al. (2004) e Ortolan (2012). Para a qualidade de vida geral, a significância estatística foi limítrofe $(p=0,07)$, apontando tendência à diferença. Contudo, essa diferença não foi constatada no que diz respeito ao bem-estar subjetivo.

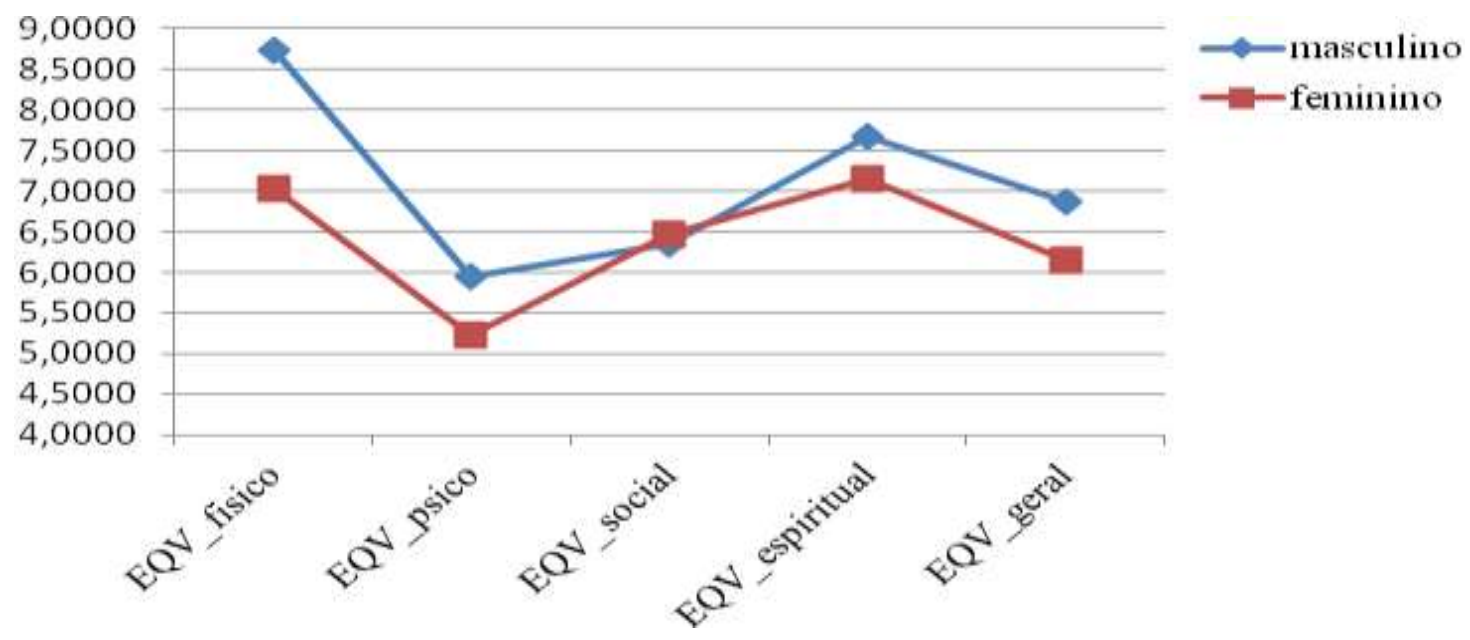

Figura 3. Avaliação dos Domínios da EQV-SC, de acordo com o Gênero dos Sobreviventes. 
Ao se comparar sobreviventes com ensino médio e sobreviventes com ensino superior, notou-se que entre estes últimos há uma melhor percepção dos domínios social e espiritual, além de aspectos positivos do bemestar subjetivo. De modo semelhante, Nagarajan et al. (2004) concluíram que uma percepção ruim de qualidade de vida associa-se a um menor nível de escolaridade.

Não foram encontradas diferenças na percepção da qualidade de vida por renda $(p>0,05)$, o que difere de outra pesquisa brasileira com sobreviventes, em que a menor renda familiar foi associada à pior avaliação da qualidade de vida no domínio físico (Ortolan, 2012). Também não foram constatadas diferenças na qualidade de vida e no bem-estar subjetivo entre sobreviventes que têm uma ocupação e sobreviventes não empregados.

Comparando-se sobreviventes com oito a 10 anos de alta com aqueles que alcançaram 11 anos ou mais, observou-se uma tendência $(p=0,056)$ de melhor qualidade de vida social por parte daqueles com mais tempo fora de tratamento.

A análise dos dados apontou correlação moderada e negativa entre a avaliação da deficiência, a percepção de qualidade de vida em geral e os domínios espiritual e social. Ou seja, quanto melhor a percepção da deficiência - por exemplo, grau leve - melhor a avaliação da qualidade de vida geral, espiritual e social. Os cálculos indicaram ainda uma correlação moderada e negativa entre percepção de deficiência e aspectos negativos e positivos. Embora haja necessidade de estudos específicos sobre deficiência e bem-estar subjetivo, reconhece-se que a deficiência pode estar associada a uma pior qualidade de vida e, em alguns casos, até mesmo a comportamentos de risco, o que pode influenciar na percepção de bem-estar (Tai et al., 2012).

As respostas às questões discursivas contidas no Questionário de Avaliação da Experiência Oncológica pelo Sobrevivente foram submetidas à análise de conteúdo temática e foram organizadas em: experiência com o câncer, tratamento e pós-tratamento (Bardin, 2010).

Muitos participantes realçaram a 'superação', na qual uma luta ou batalha teve como desfecho: suplantação de obstáculos, realização de sonhos e restabelecimento de rotinas. Seguem-se alguns trechos destes depoimentos:
"Se tiver força de vontade nada será impossível [...] tenho uma vida normal, como qualquer ser humano" (S9)

"[...] eu venci mais uma etapa, mais tem muita coisa pela frente" (S20)

"Melhorei muito como pessoa, dou mais valor à minha família, tenho bens que antes não conseguia adquirir" (S6)

'Valorização da vida após o diagnóstico' e 'finalização do tratamento' foram bastante enfatizadas pelos sobreviventes. Salci e Marcon (2010) reportam igualmente que alguns pacientes, quando em remissão da doença, desenvolvem estratégias para esquecer os riscos de um câncer, esforçando-se para pensar positivamente. Destacam-se os relatos reproduzidos a seguir:

"Após o tratamento é o período em que mais refletimos sobre a vida e o que realmente é importante para nós, o que devemos dar valor e em quem podemos contar em todas as ocasiões" (S22)

“[...] viver feliz, esquecer o passado" (S25).

'Fé e crença religiosa' foram comentadas pelos participantes. De um lado, foram enviados relatos que atribuíam significado divino ao surgimento do câncer e as dificuldades decorrentes: "[...] acredito que Deus tenha algo para nós" (S2). De outro lado, alguns relacionavam espiritualidade à continuidade da qualidade de vida e à proteção da saúde:

"Se terminou o tratamento é um presente de Deus, a fase ruim passou e em nome de Jesus não irá voltar [...] mas para Deus nada é impossível, [...] é a prova que fiquei curada totalmente" (S4)

"Primeiro, agradeci a Deus por estar vivo [...] importante estar vivo, lute, não desiste nuca [sic] você também é filhino [sic] de Deus" (S23)

"[...] procurei mais a igreja e estou com a fé fortalecida" (S6)

Tais relatos reafirmam a relevância do enfrentamento religioso/espiritual em Oncologia. Bastante investigada no cenário internacional e objeto de interesse crescente por parte de pesquisadores brasileiros, essa perspectiva conceitual tem possibilitado um debate sobre as consequências benéficas e/ou prejudiciais do uso desse tipo de recurso para lidar com as ad- 
versidades (Denney, Aten, \& Leavell, 2010; Gobatto \& Araujo, 2010; 2013; Liberato \& Macieira, 2008).

Muitos sobreviventes pareceram vivenciar uma renovação pessoal. De acordo com Stanton (2010), atributos pessoais, como otimismo e positividade, podem contribuir para esta percepção. Segundo um dos participantes:

"É um momento de extrema felicidade por estar curado e bem novamente, e poder aproveitar de forma intensa tudo o que tinha vontade de fazer durante o tratamento e não podia ou não conseguia. É uma das melhores épocas da vida, pois você se sente maravilhosamente bem e disposto a aproveitar cada segundo, cada momento da vida" (S23)

Mas, os sobreviventes mesclaram relatos de percepção positiva e negativa, o que reitera a visão de Araujo e Arrais (1998) de que a sobrevivência oncológica se caracteriza como uma vivência paradoxal.

"Após o tratamento, no começo, a adaptação é dificil, conforme o tempo passa, eu me acostumei..." (S9).

"[...] período de dificuldades, porque é necessário se readaptar às suas novas restrições e dificuldades $e$ também se readaptar a toda uma sociedade. Mas, à medida em que o tempo vai passando, vai ficando mais fácil, embora seja uma luta constante" (S13).

\section{CONCLUSÕES}

De modo geral, os sobreviventes estimaram sua qualidade de vida satisfatória, com melhor percepção do domínio físico, apesar das dificuldades enfrentadas no processo de readaptação da funcionalidade. A pior perspectiva associou-se ao domínio psicológico, ainda que mudanças positivas tenham sido reconhecidas como valorização da vida, significação da existência, aprofundamento dos laços afetivos e fortalecimento da espiritualidade. Houve discrepância entre as percepções do sobrevivente e do familiar, de modo que o familiar tendeu a perceber a qualidade de vida do sobrevivente como menos positiva, principalmente em casos de indivíduos amputados. Mas, não se verificou diferença da qualidade de vida entre sobreviventes amputados e não amputados. O escore de dor atual mostrou-se reduzido. A percepção dos efeitos colaterais da quimioterapia é negativa mesmo após o término do tratamento. A percepção de bem-estar subjetivo foi semelhante para sobreviventes e familiares. Não houve diferença quanto à idade, localização do tumor e renda entre sobreviventes.

O uso dos dois instrumentos revelou-se apropriado, pois a EQV-SC forneceu uma medida geral e de domínios específicos, enquanto a EBES completou a avaliação do domínio psicológico. A exemplo da presente pesquisa, recomendam-se investigações que adotem métodos mistos no intuito de ampliar conhecimentos acerca da etapa de sobrevivência em Oncologia. Sugere-se, igualmente, a realização de trabalhos visando à validação da Escala de Qualidade de Vida para Sobreviventes de Câncer, instrumento específico que possibilitará estudos multicêntricos no contexto brasileiro.

Por fim, diante das evidências reunidas neste trabalho, confirma-se a importância de programas destinados aos sobreviventes e seus familiares, que articulem serviços hospitalares e ações comunitárias em prol do bem-estar e da qualidade de vida da população.

\section{REFERÊNCIAS}

Ahles, T. A., Saykin, A. J., Furstenberg, C. T., Cole, B., Mott, L. A., Titus-Ernstoff, L., ... Silberfarb, P. M. (2005). Quality of life of long-term survivors of breast cancer and lymphoma treated with standard-dose chemotherapy or local therapy. Journal of Clinical Oncology, 23(19), 4399-4405.

Albuquerque, A. S., \& Tróccoli, B. T. (2004). Desenvolvimento de uma escala de bem-estar subjetivo. Psicologia: Teoria e Pesquisa, 20(2), 153-164.

Araujo, T. C. C. F., \& Arrais, A. R. (1998). A sobrevivência em oncologia: Uma vivência paradoxal. Psicologia Ciência e Profissão, 18(2), 2-9.

Bardin, L. (2010) Análise de conteúdo (4 ${ }^{\mathrm{a}}$ ed.) Lisboa: Edições 70.

Barakat, L. P., Alderfer, M. A., \& Kazak, A. E. (2006). Posttraumatic growth in adolescent survivors of cancer and their mothers and fathers. Journal of Pediatric Psychology, 31(4), 413-419.

Barr, R. D., \& Wunder, J. S. (2009). Bone and soft tissue sarcomas are often curable - but at what cost? A call to arms (and legs). Cancer, 15, 4046-4054.

Bekkering, W. P., Vlieland, T. P. M. V., Koopman, H. M., Schaap, G. R., Beishuizen, A., Anninga, J. K., ... Taminiau, A. H. M. (2012). A prospective study on quality of life and functional outcome in children and adolescents after malignant bone tumor surgery. Pediatric Blood Cancer, 58, 978-985.

Bispo Júnior, R. Z., \& Camargo, O. P. (2011). Existe diferença no prognóstico de pacientes com osteossarcoma 
primário com uma pobre resposta à quimioterapia neoadjuvante entre os graus I e II de Huvos? Revista Brasileira de Ortopedia, 46(4), 420-423.

Boaventura, C. T., \& Araujo, T. C. C. F. (2012). Estresse pós-traumático da criança sobrevivente de câncer e sua percepção acerca da experiência parental. Estudos de Psicologia (Natal), 17(2), 283-290.

Chandra, P. S., Chaturvedi, S. K., Channabasavanna, S. M., Anantha, N., Reddy, B. K. M., Sharma, S., \& Rao, S. (1998). Psychological well-being among cancer patients receiving radiotherapy - a prospective study. Quality of Life Research, 7(6), 495-500. doi: 10.1023/A:10088223 07420

Chopra, I., \& Kamal, K. M. (2012). A systematic review of quality of life instruments in long-term breast cancer survivors. Health and Quality of Life Outcomes, 10(14). Retirado de http://www.hqlo.com/content/10/1/14.

Clinton-McHarg, T., Carey, M., Sanson-Fisher, R., Shakeshaft, A., \& Rainbird, K. (2010). Measuring the psychosocial health of adolescent and young adult (AYA) cancer survivors: A critical review. Health and Quality of Life Outcomes, 8(25), 1-13.

Costa, L. S. M., \& Pereira, C. A. A. (2007). Bem-estar subjetivo: Aspectos conceituais. Arquivos Brasileiros de Psicologia, 59(1), 72-80. Retirado de http://pepsic.bvsa lud.org/scielo.php?script=sci_arttext\&pid=S1809-52672 007000100008\&lng=pt\&nrm=iso

Costa Neto, S. B., \& Araujo, T. C. C. F. (2008). Qualidade de vida do enfermo oncológico: Um panorama sobre o campo e suas formas de avaliação. Em V. A. Carvalho, M. H. P. Franco, M. J. Kovács, R. Liberato, M. T. Macieira, M. J. B. Gomes \& L. Holtz (Eds.), Temas em Psico-oncologia (pp. 195-208). São Paulo: Summus.

Delgado-Sanz, M. C., García-Mendizábal, M. J., Pollán, M., Forjaz, M. J., López-Abente, G., Aragonés, N., \& Pérez-Gómez, B. (2011). Health-related quality of life in Spanish breast cancer patients: A systematic review. Health and Quality of Life Outcomes, 9(3), 1-10.

Demark-Wahnefried, W. (2010). Changing health behaviors after treatment. Em J. C. Holland, W. S. Breitbart, P. B. Jacobsen, M. S. Lederberg, M. J. Loscalzo, \& R. McCorle (Eds.), Psycho-Oncology (pp. 551-556). New York: Oxford University Press.

Denney, R. M., Aten, J. D., \& Leavell, K. (2010). Posttraumatic spiritual growth: A phenomenological study of cancer survivors. Mental Health, Religion \& Culture, 14(4), 371-391.

Diener, E., Emmons, R. A., Larsen, R. J., \& Griffin, S. (1985). The satisfaction with life scale. Journal of Personality Assessment, 49, 71-75.

Eiser, C. (2009). Assessment of health-related quality of life after bone cancer in young people: Easier said than done. European Journal of Cancer, 45(10), 1744-1747.
Ersek, M., Ferrell, B. R., Hassey-Dow, K., \& Melancon, C. H. (1997). Quality of life in women with ovarian cancer. Western Journal of Nursing Research, 19(3), 334-350. doi: 10.1177/019394599701900305

Fleck, M. P. A. (Ed.). (2008). A avaliação da qualidade de vida. Guia para profissionais de saúde. Porto Alegre: Artmed.

Frances, J. M., Morris, C. D., Arkader, A., Nikolic, Z. G., \& Healey, J. H. (2007). What is quality of life in children with bone sarcoma? Clinical Orthopaedics and Related Research, 459, 34-39.

Furst, L., \& Humphrey, M. (1983). Coping with the loss of a leg. Prosthetics and Orthotics International, 7(3), 52156.

Ganz, P. A., \& Hahn, E. E. (2010). Implementing the survivorship care plan: A strategy for improving the quality of care for cancer survivor. In J. C. Holland, W. S. Breitbart, P. B. Jacobsen, M. S. Lederberg, M. J. Loscalzo \& R. McCorle (Eds.), Psycho-Oncology (pp. 557561). New York: Oxford University Press.

Giacomoni, C. H. (2004). Bem-estar subjetivo: Em busca da qualidade de vida. Temas em Psicologia da Sociedade Brasileira de Psicologia, 12(1), 43-50.

Gobatto, C. A., \& Araujo, T. C. C. F. (2013). Religiosidade e espiritualidade em oncologia: Concepções de profissionais da saúde. Psicologia USP, 24(1), 11-34.

Gobatto, C. A., \& Araujo, T. C. C. F. (2010). Coping religioso-espiritual: Reflexões e perspectivas para a atuação do psicólogo em oncologia. Revista da Sociedade Brasileira de Psicologia Hospitalar, 13(1),52-63.

Holland, J. C., \& Reznik, I. (2005). Pathways for psychosocial care of cancer survivors. Cancer Supplement, 104(11), 2624-2637. doi:10.1002/cncr.21252

Instituto Nacional do Câncer. (2008). Câncer da criança e adolescente no Brasil: Dados dos registros de base populacional e de mortalidade. Rio de Janeiro: INCA.

Jagannathan, A., \& Juvva, S. (2009). Life after cancer in India: Coping with side effects and cancer pain. Journal of Psychosocial Oncology, 27, 344-360.

Kazak, A. E., Alderfer, M. A., Rourke, M. T., Simms, S., Streisand, R., \& Grossman, J. R. (2004). Posttraumatic stress disorder (PTSD) and posttraumatic stress symptoms in families of childhood cancer survivors. Journal of Pediatric Psychology, 29(3), 211-219.

Khan, N. F., Rose, P. W., \& Evans, J. (2012). Defining cancer survivorship: A more transparent approach is needed. Journal of Cancer Survivorship, 6, 33-36.

Kim, Y., Carver, C. S., Spillers, R. L., Crammer, C., \& Zhou, E. S. (2011). Individual and dyadic relations between spiritual well-being and quality of life among cancer survivors and their spousal caregivers. Psycho-Oncology, 20, 762-770. 
Klosky, J. L., \& Spunt, S. L. (2010). Sarcoma. Em J. C. Holland, W. S. Breitbart, P. B. Jacobsen, M. S. Lederberg, M. J. Loscalzo \& R. McCorle (Eds.), PsychoOncology (pp. 187-190). New York: Oxford University Press.

Koopman, H. M., Koetsier, J. A., Taminiau, A. H. M., Hijnen, K. E., Bresters, D., \& Egeler, R. M. (2005). Health-related quality of life and coping strategies of children after treatment of a malignant bone tumor: A 5year follow-up study. Pediatric Blood Cancer, 45, 694699.

Lawrence, R. H., \& Liang, J. (1988). Structural integration of the affect balance scale and the life satisfaction index A: Race, sex, and age differences. Psychology and Aging, 3, 375-384.

Liberato, R. P., \& Macieira, R. C. (2008). Espiritualidade no enfrentamento do câncer. In V. A. Carvalho, M. H. P. Franco, M. J. Kovács, R. Liberato, M. T. Macieira, M. J. B. Gomes \& L. Holtz (Eds.), Temas em Psico-oncologia (pp. 414-431). São Paulo: Summus.

Maurice-Stam, H., Grotenhuis, M. A., Caron, H. N., \& Last, B. F. (2006). Quality of life and current coping in young adult survivors of childhood cancer: Positive expectations about the further course of the disease were correlated with better quality of life. Psycho-Oncology, 15(1), 31-43.

Maurice-Stam, H., Oort, F. J., Last, B. F., \& Grotenhuis, M. A. (2009). A predictive model of health-related quality of life in young adult survivors of childhood cancer. $E u$ ropean Journal of Cancer Care, 18(4), 339-349.

Morganstern, B. A., Bochner, B., Dalbagni, G., Shabsigh, A., \& Rapkin, B. (2011). The psychological context of quality of life: A psychometric analysis of a novel idiographic measure of bladder cancer patients' personal goals and concerns prior to surgery. Health and Quality of Life Outcomes, 9(10), 1-18.

Nagarajan, R., Clohisy, D. R., Neglia, J. P., Yasui, Y., Mitby, P.A, Sklar, C., ... Robison, L. L. (2004). Function and quality of life of survivor of pelvic and lower extremity osteossarcoma and Ewing's sarcoma: The childhood cancer survivor study. British Journal of Cancer, 91(11), 1858-1865.

Nagarajan, R., Kamruzzaman, A., Ness, K. K., Marchese, V. G., Sklar, C., Mertens, A., ... Marina, N. (2011). Twenty years of follow-up of survivors of childhood osteosarcoma - a report from the childhood cancer survivor study. Cancer, 117(3), 625-634.

Nagarajan, R., Neglia, J. P., Clohisy, D. R., Yasui, Y., Greenberg, M., Hudson, M., ... Robison, L. L. (2003). Education, employment, insurance, and marital status among 694 survivors of pediatric lower extremity bone tumors - a report from the childhood cancer survivor study. Cancer, 97(10), 2554-2564.
Ness, K. K., Bhaskar, P. T., Rao, B. N., \& Daw, N. C. (2009). More than physical function: HRQOL among children and adolescents with lower extremity bone sarcoma. Pediatric Blood Cancer, 53(3), 310-311.

Nucci, A. G., \& Valle, E. R. M. (2006). Quality of life and cancer. Applied Cancer Research, 26(2), 66-72.

Ortolan, P. E. (2012). Qualidade de vida do adulto jovem sobrevivente de leucemia linfóide aguda pediátrica. Dissertação de Mestrado. Universidade Estadual de Campinas, Campinas.

Patrick, D. L. (2008). A qualidade de vida pode ser medida? Como? Em M. R. A. Fleck. A avaliação da qualidade de vida: guia para profissionais de saúde (pp. 29-39). Porto Alegre: Artmed.

Pearce, N. J. M., Sanson-Fisher, R., \& Campbell, H. S. (2008). Measuring quality of life in cancer survivors: A methodological review of existing scales. PsychoOncology, 17(7), 629-640.

Perina, E. A., Mastellaro, M. J., \& Nucci, N. A. G. (2008). Efeitos tardios do tratamento do câncer na infância e na adolescência. Em V. A. Carvalho, M. H. P. Franco, M. J. Kovács, R. Liberato, M. T. Macieira, M. J. B. Gomes \& L. Holtz (Eds.), Temas em Psico-oncologia (pp. 414431). São Paulo: Summus Editora.

Petrilli, A. S. (2008). Câncer ósseo. Em V. A. Carvalho, M. H. P. Franco, M. J. Kovács, R. Liberato, M. T. Macieira, M. J. B. Gomes \& L. Holtz (Eds.), Temas em Psicooncologia (pp. 120-129). São Paulo: Summus Editora.

Salci, M. A., \& Marcon, S. S. (2010). A convivência com o fantasma do câncer. Revista Gaúcha de Enfermagem, $31(1), 18-25$.

Santos, A. L. A., Moura, J. F. P., Santos, C. A. A. L., Figueiroa, J. N., \& Souza, A. I. (2012). Avaliação da qualidade de vida relacionada à saúde em pacientes com câncer do colo do útero em tratamento radioterápico. $R e$ vista Brasileira de Cancerologia, 58(3), 507-515.

Schwartz, L. A., Werba, B. E., \& Kazak, A. E. (2010). Adult survivors of childhood cancer. Em J. C. Holland, W. S. Breitbart, P. B. Jacobsen, M. S. Lederberg, M. J. Loscalzo \& R. McCorle (Eds.), Psycho-Oncology (pp. 562-566). Oxford University Press.

Silva, C. H. D., \& Derchain, S. F. M. (2006). Qualidade de vida em mulheres com câncer ginecológico: Uma revisão de literatura. Revista Brasileira de Cancerologia, 52(1), 33-47.

Siqueira, M. M. M., \& Padovam, V. A. R. (2008). Bases teóricas de bem-estar subjetivo, bem-estar psicológico e bem-estar no trabalho. Psicologia: Teoria e Pesquisa, 24(2), 201-220.

Stanton, A. L. (2010). Positive consequences of the experience of cancer: Perceptions of growth and meaning. Em J. C. Holland, W. S. Breitbart, P. B. Jacobsen, M. S. Le- 